\title{
Error Detection and Classification of Tangential Radial Single Machining of Non-Circular Gear
}

\author{
Jianqing Dai, Shengze Wang \\ School of mechanical engineering, Donghua University, Shanghai 201620, People's Republic of China
}

\begin{abstract}
The studies on non-circular gear cutting and using errors were generally based on theory of cylindrical gear errors, and the similarities and differences between cylindrical gear and non-circular gear were combined to study the change rule of non-circular gear contacting line and obtained corresponding conclusions. In this paper, from a different perspective, by starting from the non-circular gear engagement principle, on the basis of analyzing the concrete geometric error conditions, new type tooth profile algorithm routine is reasonably modified, and needed noncircular gear tooth profile error contrast figure is obtained. Furthermore, theoretical derivation is carried out by incorporating analytical method and contacting line incremental method, validity of computer simulation is verified, a reasonable classification method is proposed based on the characterization of the error characteristic code in the general case, Summed up the error genetic map which possess enormous significance to direct practice generation[1].
\end{abstract}

\section{Introduction}

Non-circular gear in terms of its essence can be considered as a new type of institution which integrates the advantages of both cam and gear. Non-circular gear mechanism not only inherited the numerous congenital advantage of the gear mechanism, but also has predictable characteristics of cam mechanism transmission rule; therefore the non-circular gear is a kind of gear mechanism can achieve predictable variable speed ratio transmission [2], [3]. After years of research and development, non-circular gear foundation design theory has been basically complete, but now the application scope of the non-circular gear and its inherent advantage doesn't match. The reason lies in the rather difficult manufacturing, the researches in manufacturing lack systematicness and completeness, especially that the standard of non-circular gear machining error has not been set, which greatly restricted the speed that noncircular gear developed from theory to practical application [4].

Non-circular gear machining exist unique errors, respectively are the tool setting error [5], [6], the main reason of tool setting error is the human factor, and the error size is far greater than other sources of error. In the actual statistics tool setting error accounted for noncircular gear machining error causes up to about 70\% [7], through the tool setting error analysis method can also be used in other areas of error, so the study on tool setting error has very important practical significance.

\section{Error model}

According to the principle of gear meshing, we can get the model of tangential and radial error. In tangential, radial and geometric eccentricity errors, Tangential and radial errors change the meshing position of pitch curve, which will change the shape of tooth profile curve, so we studied respectively with tangential error and radial error.

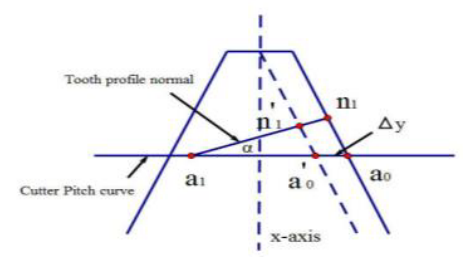

Figure 1. Tangential error model [1]

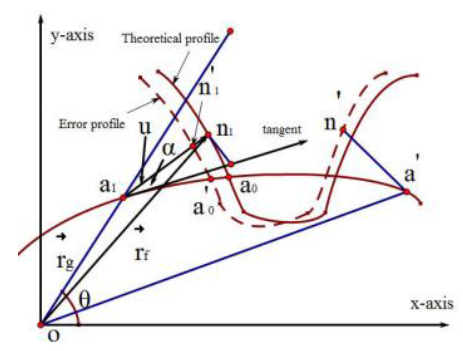

Figure 2. Tangential profile analytic graph of non-circular gear

As shown in Fig. 1[1], zero position of serrateknife just aligned the collinear with the zeroposition of workpiece at a time of zero, in actual production, because of the existence of tool setting error; the tooth cutter has $\Delta y$ offset in the axial direction. The length of vector in Fig. 1 will cause $\overrightarrow{a_{1} n_{1}}$ to $\overrightarrow{a_{1} n_{1}^{\prime}}$, the tooth profile normal in Fig. 3. will cause $\overrightarrow{a_{1} n_{1}}$ to $\overrightarrow{a_{1}^{\prime} n_{1}}$. 


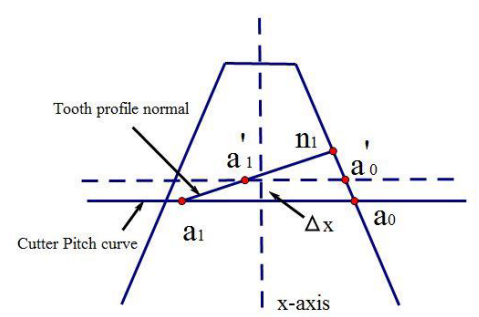

Figure 3. Radial error model

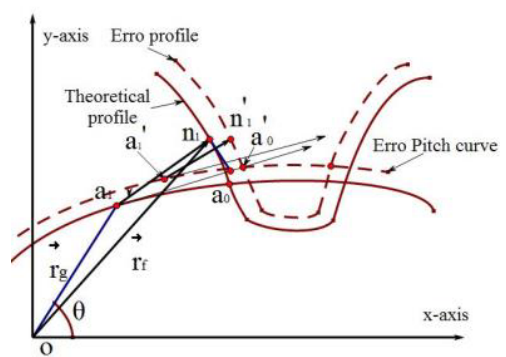

Figure 4. Radial profile analytic graph of non-circular gear

\section{Computer simulation and graphic representation}

We used beforehand programmed non-circular gear tooth profile algorithm program, the program's main algorithm idea is shown in Fig. 5 first of all, we carried out a generative simulation of the serrated knife according to the operation condition of the zero alignment, and we got the theory tooth profile diagram without the error, then we add a fixed parameter error in the $x$ coordinate value of serrated knife small coordinate system to the subroutine to use the error offset in Fig. 1, he initial position of the $x$ axis in the coordinate system is parallel to the direction of the $y$ axis, in order to simulate the tool setting error condition. Then the program runs again to get the tooth profile pattern when the tool setting error appeared. Finally, two pictures were combined in the same coordinate system to form Fig. 1[1].

Fig. 6(a) and Fig. 6(c) shows the positive direction and negative direction of long axis of tooth profile comparison chart of cogging under the tool setting error, Fig. 6(b) and Fig. 6(d) shows the positive direction and negative direction of short axis of tooth profile comparison chart of cogging under the tool setting error. Small red circles shows the theory tooth profile without error in Fig. 6, while blue stars are tooth profile graphs that have the tool setting error.

From Fig. 6 we can see

(1) The left and right profile of the error profile is in the same direction as the theoretical profile.

(2) The formed tooth profile and the theory tooth profile after the excursion of tooth profile contrast diagrams are different, that is, error tooth profile isn't the simple equidistant line of theory tooth profile.

(3) At the bottom of the Error profile and theoretical profile of maintain level in normal direction, that is, the radial error is zero.

From Fig. 7 we can see

(4) The positive displacement in Fig. $7(a)$ is offset along the normal direction, the positive displacement in Fig. $7(b)$ is offset along the negative direction of the normal.

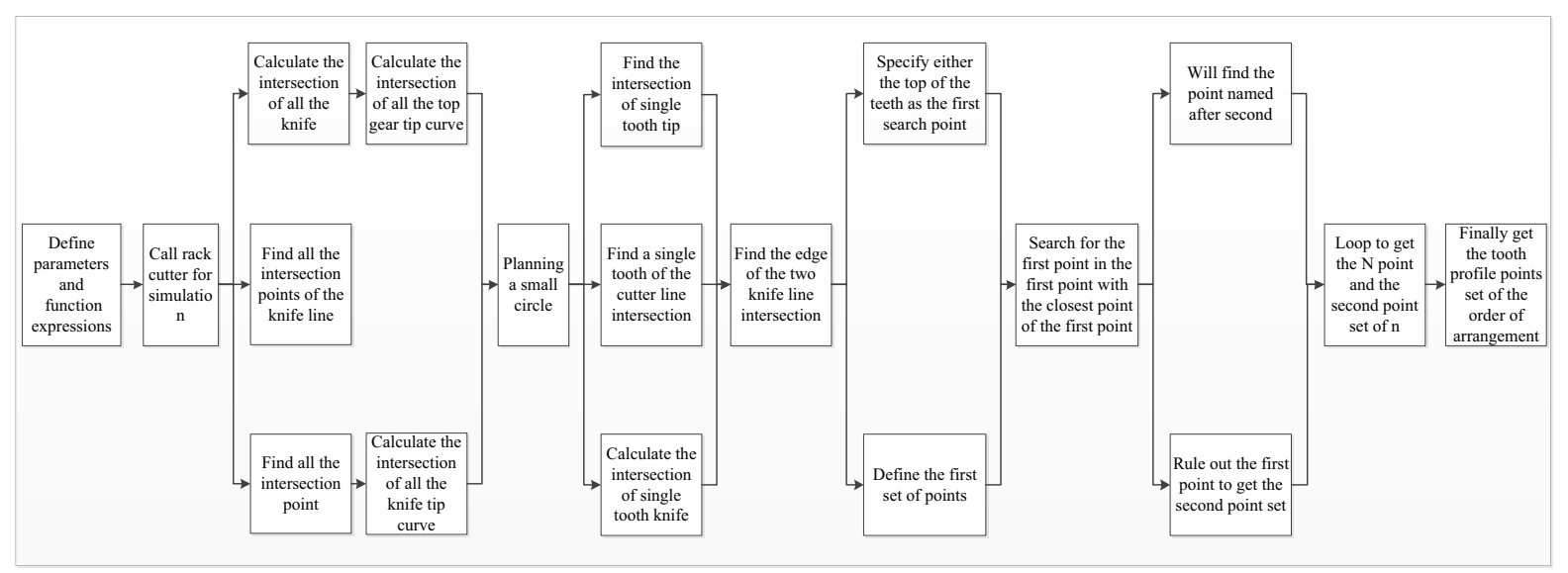

Figure 5. Algorithm flow

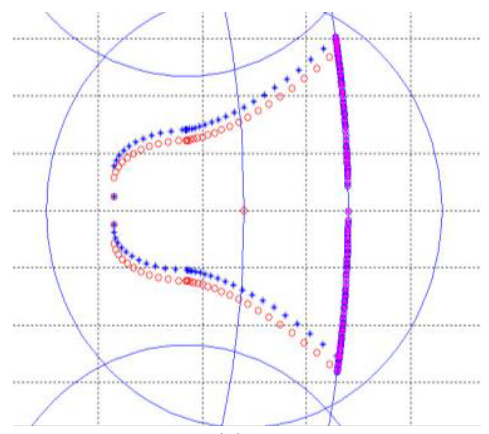

(a)

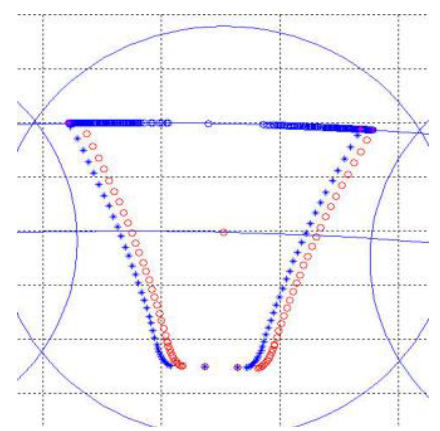

(b)

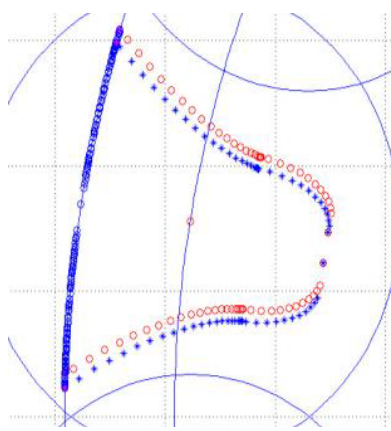

(c)

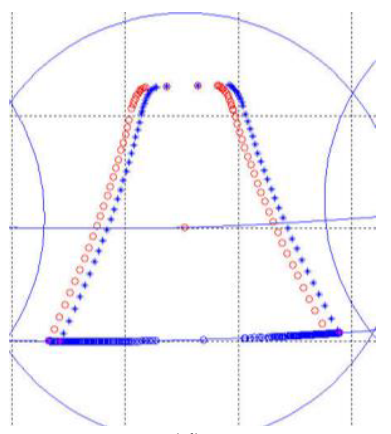

(d)

Figure 6. Tangential error 


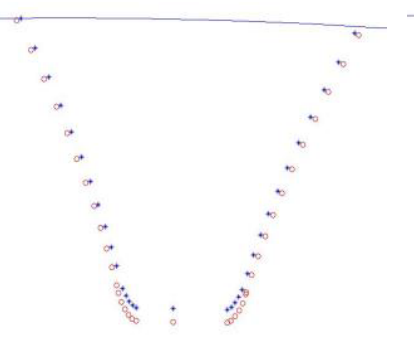

(a)

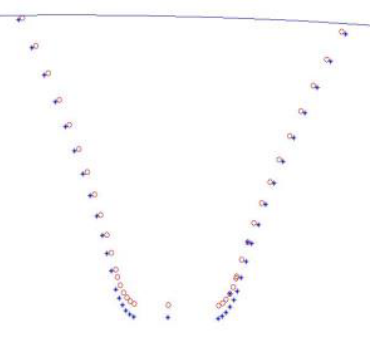

(b)
Figure7. Radial error

\section{Theoretical verification and analysis}

\subsection{Theoretical verification [1]}

In order to set the tooth profile normal distribution regulation of the non-circular beforehand for convenience, it's determined by the following [8].

$$
\lambda-\mu=\text { constant }
$$

From Fig. 1 we can see

$$
\begin{gathered}
a_{1} n_{1}=a_{0} a_{1} * \cos \alpha \\
S_{1}=a_{0} a_{1}
\end{gathered}
$$

The serrated knife excursions also comply with the above rule, but the arc length changes. In Fig. 2, vector equation of the tooth profile can be expressed by the following $[9,10]$.

$$
\overrightarrow{r_{f}}=\overrightarrow{r_{g}}+\overrightarrow{a n}
$$

Right tooth profile equationis obtained when converting into rectangular coordinates.

$$
\left\{\begin{array}{l}
x_{R}=r_{g} \cos \theta+\text { an } \cos (\theta+u-\alpha) \\
y_{R}=r_{g} \sin \theta+\text { an } \sin (\theta+u-\alpha)
\end{array}\right.
$$

Left tooth profile equation

$$
\left\{\begin{array}{l}
x_{L}=r_{g} \cos \theta+a^{\prime} n^{\prime} \cos (\theta+u+\alpha-\pi) \\
y_{L}=r_{g} \sin \theta+a^{\prime} n^{\prime} \sin (\theta+u+\alpha-\pi)
\end{array}\right.
$$

The tooth profile normal length is acquired by the following

$$
\begin{aligned}
& a n=a_{0} a \cos \alpha_{u}=S * \cos \alpha_{u} \\
& a n=a_{0} a \cos \alpha_{u}=S * \cos \alpha_{u}
\end{aligned}
$$

The analytical formula for theory tooth profile is above one offset $\Delta y$ in Fig. 1 is as following after theory tooth profile.

$$
a_{0} a_{0}^{\prime}=\Delta y
$$

Simplified as error vector quantity

$$
a_{1} n_{1}^{\prime}=a_{1} n_{1}-a_{0} a_{0}^{\prime} \cos \alpha_{u}
$$

Formula is got after being converted into rectangular coordinate.

$$
\left\{\begin{array}{l}
x_{n}^{\prime}=x_{n} \mp a_{0} a_{0}^{\prime} \cos \alpha_{u} \operatorname{tg} \alpha_{u} \\
y_{n}^{\prime}=y_{n} \mp a_{0} a_{0}^{\prime} \cos \alpha_{u} \operatorname{ctg} \alpha_{u}
\end{array}\right.
$$

In the traditional meshing line increment method of cylindrical gear tooth profile error analysis method [9]:

$$
\Delta F=\sum_{k=1}^{n} e_{k} \sin \left(K \varphi+\varphi_{k}\right)
$$

$e_{k}$ is eccentric error of gear, $K$ is gear error frequency in a turn, $\varphi$ is corner, $\varphi_{k}$ is phase angle.

$$
\begin{gathered}
\Delta r_{r e}=\frac{\Delta F_{\text {left }}+\Delta F_{\text {right }}}{2 \sin \alpha} \\
\left\{\begin{array}{l}
\Delta F_{\text {runleft }}=+e_{\text {run }} \sin \varphi \\
\Delta F_{\text {runright }}=-e_{\text {run }} \sin \varphi
\end{array}\right.
\end{gathered}
$$

Bring Eq. (14) into Eq. (13) to obtain radial direction error equation

$$
\Delta r=\frac{e_{r u n} \sin \varphi-e_{r u n} \sin \varphi}{2 \sin \alpha}
$$

In accordance with the tangential error theory, it is also shown that the tooth profile of the Fig. 4 theory and the position of the generating error:

$$
\begin{gathered}
a_{1} n_{1}=a_{0} a_{1} * \cos \alpha \\
a_{1}^{\prime} n_{1}^{\prime}=a_{0}^{\prime} a_{1}^{\prime} * \cos \alpha \\
a_{0}^{\prime} a_{1}^{\prime}=a_{0} a_{1}-\frac{\Delta x(\sin \alpha+\cos \alpha)}{\sin \alpha}
\end{gathered}
$$

According to the meshing principle, the instantaneous turning center of the gear cutter is $a_{1}$ point, The $n_{1}$ points on the tooth profile of the rack are corresponding to the $n$ points on the conjugate teeth, When the gear cutter is up to $\Delta x, a_{1}$ points and $a_{0}$ were turned into graphics in the $a_{1}^{\prime}$ point and $a_{0}^{\prime}$. The remaining part of the use of the formula in front of the $4 \sim 6$ that can be obtained $n_{1}$ and $n_{1}^{\prime}$ coordinates of the position.

\subsection{Theoretical analysis}

In Fig. 1[1], the $\Delta y$ causes the $a_{0}$ to move to the $a_{0}^{\prime}$ position, causing the $\overrightarrow{a_{1} n_{1}^{\prime}}<\overrightarrow{a_{1} n_{1}}$. In the Fig. 6 and Eq. (4) 
an shorter makes the error profile of the left tooth profile to the counter clockwise direction, In the same way, the an of the right tooth profile makes the error profile to the same direction as the right tooth profile, This explains the reason for the graphical representation. By Eq. (15), we know due to the various curvature radiuses of points on the section curve, leading to the each included angles $\mu$ of each point radius vector and the tangent line are not identical, the vectorial direction of the offset is not exactly the same, so it can be proved the correctness of the graphical representation. The reason that radial error does not appear can be got by Eq. (15).

Promotion to general situation, we can get the tool clamping error is actual a specially case of variable tangential direction error. Tool clamping error is got when $\Delta y$ is a constant value, when $\Delta y$ is a variate value, in principle, we need to determine the relevant $\Delta y$ value that each gear cutting angle of tooth profile should correspond with, and consider the variate value $\Delta y$ as the serrated knife offset of corresponding angle.

In variable tangential direction error conditions, offset $\Delta y$ changes follow the change of the cutting angle and comply with the sine function law $\Delta y=k \sin \theta$.

In the radial error contrast diagram, in the same way by formula $16 \sim 18$ and formula $4 \sim 6$, we can obtain that the radial error profile is not the line of the theoretical profile.

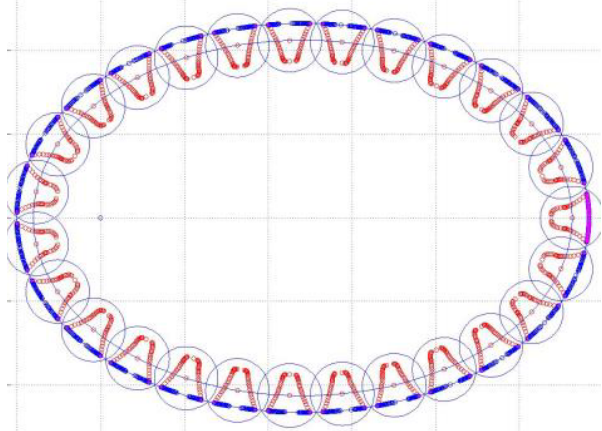

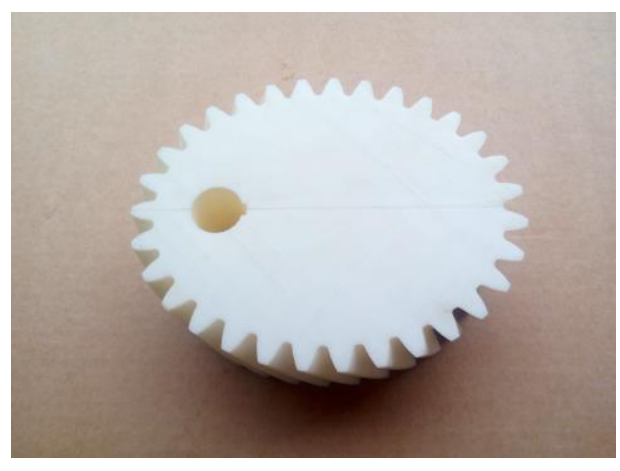

Figure 9. Solid model of non-circular gea

\section{Experiment and classification}

Using the preceding algorithm program we take the most common elliptic gear as an example. Fig. 8. for elliptic gear tooth profile, and we can process the non-circular gear solid model which is shown in Fig. 9. through the rapid prototyping system,Using the RoyalArm RA315 measuring arm (measurement accuracy of $0.05 \mathrm{~mm}$ ) which shows in Fig. 10. on the key points of non-circular gear contrate profile actual coordinates value and theoretical coordinate value difference to determine what kind of error [1].

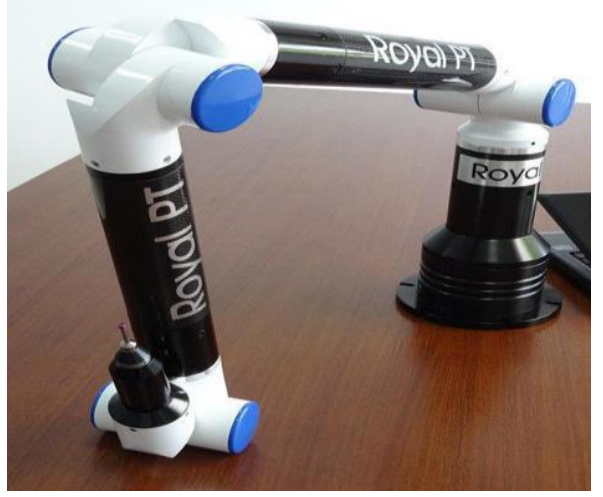

Figure 10. Joint arm measuring instrument

Figure 8. elliptic non-circular gear tooth profile

Table 1. Data sheet[1]

\begin{tabular}{|c|c|c|c|c|c|c|c|c|}
\hline & \multicolumn{2}{|c|}{$\begin{array}{l}\text { Long axis normal } \\
\text { direction }\end{array}$} & \multicolumn{2}{|c|}{$\begin{array}{l}\text { Short axis normal } \\
\text { direction }\end{array}$} & \multicolumn{2}{|c|}{$\begin{array}{l}\text { Long axis negative } \\
\text { direction }\end{array}$} & \multicolumn{2}{|c|}{$\begin{array}{l}\text { Short axis negative } \\
\text { direction }\end{array}$} \\
\hline $\begin{array}{l}\text { X- theoretical tooth } \\
\text { top apex }\end{array}$ & 35.2954 & 35.2954 & 16.7847 & 14.3307 & -5.4062 & -5.9078 & 14.3250 & 16.7847 \\
\hline $\begin{array}{l}\text { Y- theoretical tooth } \\
\text { top apex }\end{array}$ & -1.4003 & 1.4003 & 13.9980 & 13.9331 & 3.0883 & 0.3393 & -13.9980 & -13.9330 \\
\hline $\begin{array}{l}\text { X-actual tooth top } \\
\text { apex }\end{array}$ & 35.30 & 35.30 & 16.70 & 14.45 & -5.40 & -5.90 & 14.40 & 16.90 \\
\hline $\begin{array}{l}\text { Y- actual tooth top } \\
\text { apex }\end{array}$ & 1.30 & 1.45 & 13.95 & 14.00 & 3.00 & 0.25 & -14.00 & -13.95 \\
\hline $\begin{array}{l}\mathrm{X} \text { - theoretical } \\
\text { tooth of root }\end{array}$ & \multicolumn{2}{|c|}{33.0952} & \multicolumn{2}{|c|}{15.4782} & \multicolumn{2}{|c|}{-3.5371} & \multicolumn{2}{|c|}{15.4745} \\
\hline $\begin{array}{l}\text { Y- theoretical } \\
\text { tooth of root }\end{array}$ & \multicolumn{2}{|c|}{0} & \multicolumn{2}{|c|}{11.7433} & \multicolumn{2}{|c|}{1.3606} & \multicolumn{2}{|c|}{-11.7436} \\
\hline $\begin{array}{c}\mathrm{X}-\text { actual } \\
\text { tooth of root }\end{array}$ & \multicolumn{2}{|c|}{33.10} & \multicolumn{2}{|c|}{15.40} & \multicolumn{2}{|c|}{-3.55} & \multicolumn{2}{|c|}{15.55} \\
\hline $\begin{array}{c}\text { Y-actual } \\
\text { tooth of root }\end{array}$ & \multicolumn{2}{|c|}{0.10} & \multicolumn{2}{|c|}{11.75} & \multicolumn{2}{|c|}{1.25} & \multicolumn{2}{|c|}{-11.70} \\
\hline
\end{tabular}



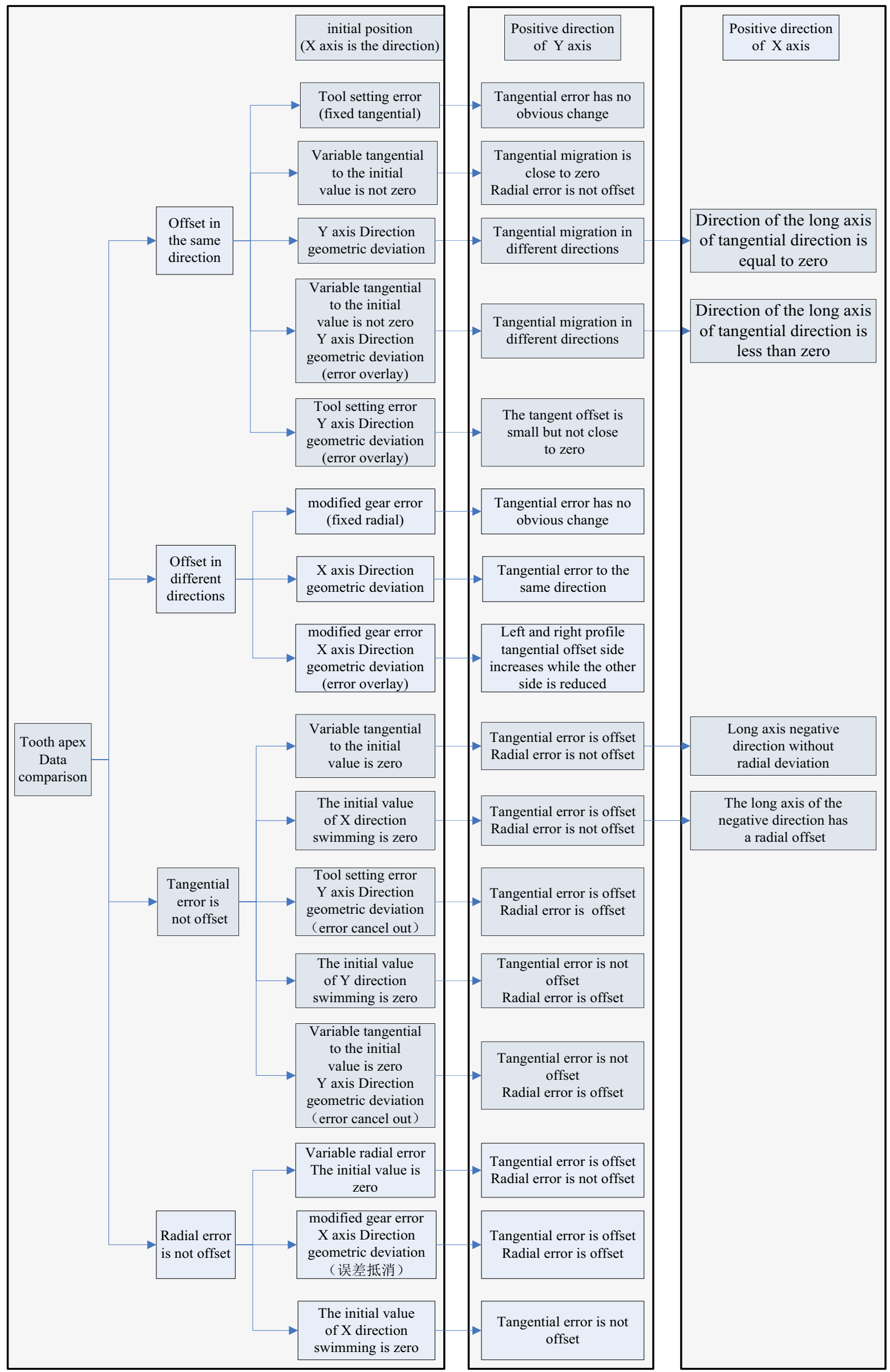

Figure 11. Characterization of characteristic code 


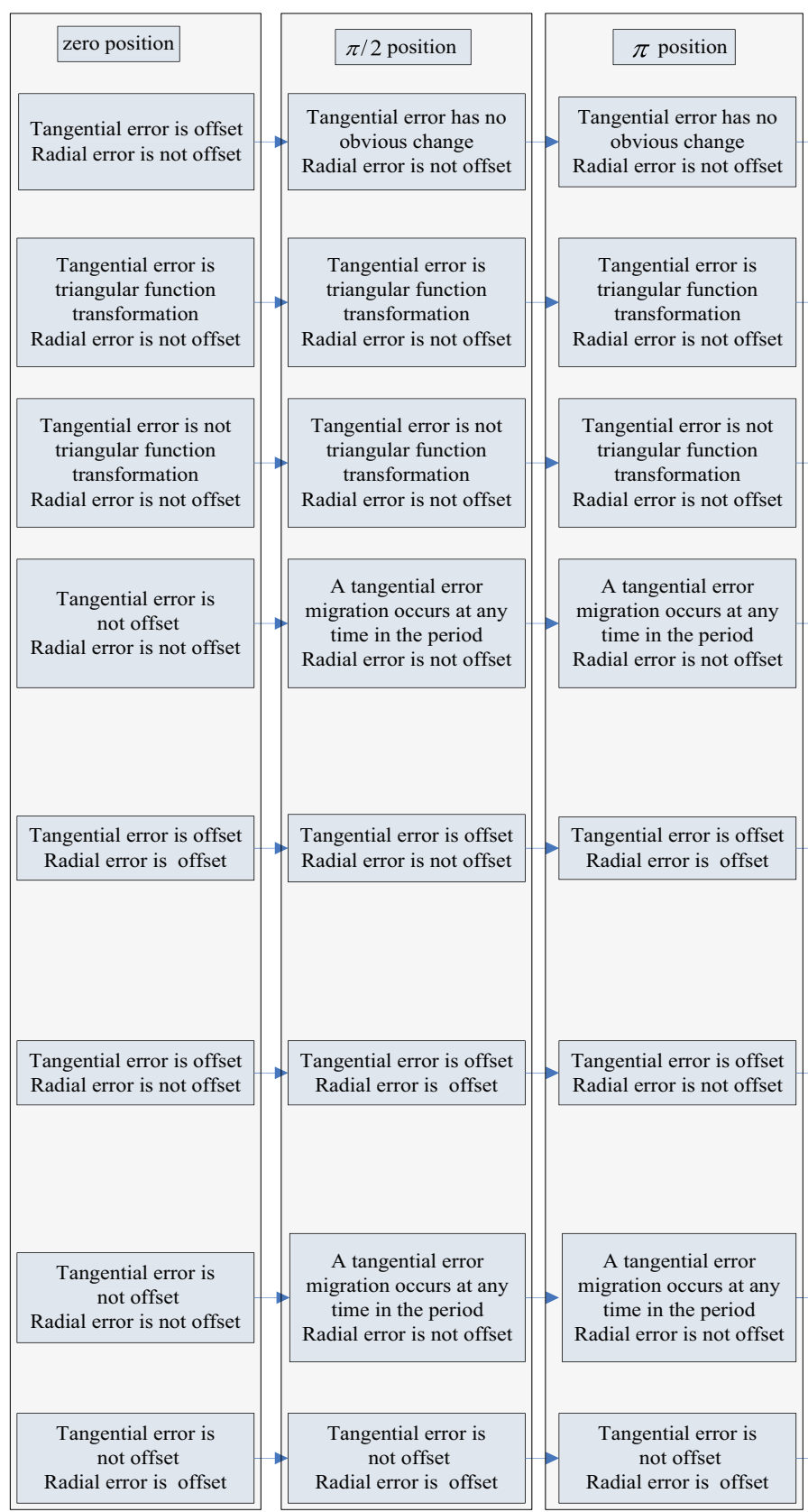

Figure 12. error factors of error genetic diagr

The experimental method is as follows [1].

(1) Non-circular gear processing must have a zero, that is, the initial processing bit. In the model above the left end elliptic focus as the origin of coordinates, the right level for the positive direction of axis, vertical axis direction, establish coordinate system.

(2) Because of the large number of non-circular gear tooth, large quantities of one of the measurement of each tooth space for all positions of the geometric point in the actual production is not acceptable, so we at the end of the ellipse long axis and short axis at both ends of each selected a tooth, coordinate measurement of tooth top apex and tooth root value.

(3) The actual coordinates the collection value and coordinate theory are compared to the values, the zero cogging data must be collected, the cases of zero cogging in long axis are the direction and the results are shown in Table I.

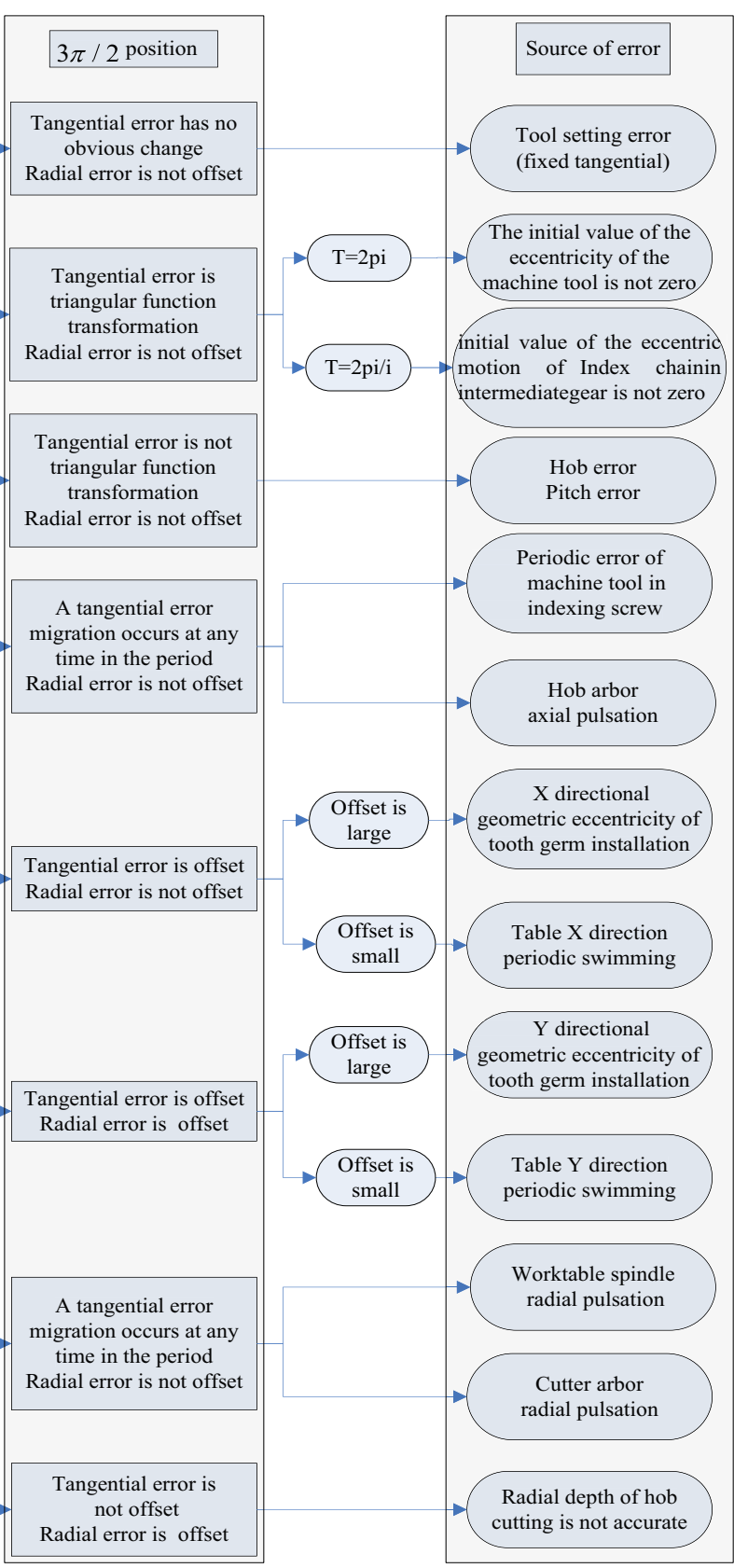

(4) According to the results of the comparison of the results in Fig. 11 can be classified to learn about the causes and sources of error. Because this article is only relates to cut to the field of error, Fig. 11 relates to the radial error and the geometric deviation theory proof in my other are published articles are presented in detail. This only references related to cut to the part of the conclusion of the error. In Table I at the coordinate data of zero tooth apex the conclusion can be obtained that the tooth profile is not offset, and the tangential direction is shifted in the same direction., From Fig. 11 we know this phenomenon may have five reasons: tool setting error, cut to the initial error is not zero, the direction of geometrical deviations, cut to the initial error is zero and the direction of the geometric deviation of these two factors superimposed, tool setting error and the orientation geometric deviation of these two factors superimposed. The data of the short axis normal direction 
can be obtained in the radial direction, and there is no significant change in the tangential migration, from Fig. 11 we know the model belongs to the typical causes of tool setting error.

From this example we get the test method of the origin of the error and the characterization of the genetic code. In the actual production we are more concerned about how to quickly troubleshoot what are the process error factors that cause the error, so in Fig. 11 based combined with common cause of cut to error of process factors are summarized Fig. 12 non-circular gear error factors of the error genetic diagram. So in Fig. 11 based on combination of common cause of cutting error of process factors to get Fig. 12 non-circular gear error factors of the error genetic diagram. This will provide a powerful tool for the production of the cause of the problem quickly.

\section{Innovation andweaknesses}

Via the system analysis and the theoretical proof of the computer simulation results for non-circular gear tool setting error, we may obtain the conclusion that the tool setting error is a fixed variable tangential error, it's beneficial for the research complement of the noncircular gear error system and lay a solid foundation for the subsequent individual error of other conditions, and got the condition code of the tool setting error excursion, providing theory support for timely finding out the cause of the gear error when the factory inspection, thus improve the machining precision of the non-circular gear [1].

At the same time, we must realize that although all the errors mentioned above can be attributed to the error of tangential, radial and geometric errors, the error can be formed separately or in combination. But the source of the process error is different, and the combination of various errors is more complicated. So the Fig. 11 and Fig. 12 summarizes the graphical representation and the error factors are not one-to-one relationship, actual operation must be flexible use must not be applied mechanically, the geometric errors and the cycle of variation of the machine tool swimming factor in Fig. 11 and Fig. 12 can be guaranteed by $T=2 \pi$, but you need to get more data points with changes to determine the cause of the error when other $T \neq 2 \pi$ Error period. In single error or simple two kinds of combination of errors when the method has accurate recognition ability, when the superposition of many error the method also has certain reference value, so it seems the method also has a lot of room for improvement.

\section{References}

1. Jianqing Dai,Shenzhe Wang, in:Analogue simulation and analysis of non-circular gear reentry error, ICMMR2016

2. F.L.Litvin. Generation of planar and helical elliptical gears by application of rack- cutter, hob, and shaper, 196:4321-4336. Comput. Methods Appl. Mech. Engrg, (2007).

3. Guoxing Sun, Chuanqiong Sun, Yongde Liu, in: The application prospect of non-circular gear on infinitely variable speed, 1: 1-4. Equipment maintenance technology, (2009).

4. Xueqiang Gao, in: Transmission technology overview of non-circular gear, 3:5-8. Mechanical drive, (2003).

5. Huiping Yan, Chibing $\mathrm{Hu}$, Limei Liu, in: The research of straight tooth non-circular gear hobbing machining error analysis, 4: 18-20.Mechanical drive, (2007).

6. Fangyong Tian, Chibing Hu, Yancang Jiang, in: An automatic tool setting method of non-circular gear hobbing, 4: 41-43 [J].Mechanical research and application, (2010).

7. Liqun $\mathrm{Wu}$, Hongxia $\mathrm{Bi}$, in: The determination of axial special-shaped gear NC hobbing machining adjusted cutter point coordinates, 6:109-112.Coal mine machinery, (2008).

8. Yongping Liu, Peng Wang, Jia Li, in: The elliptical gear tooth profile design via analytical method, 2: 214-216. Machinery design and Manufacture, (2015).

9. Fusheng Li. Non-circular gear, (1975).

10. Mingtong Wang. Involute gear accuracy, (1983). 